\title{
Genome-wide association analyses reveal significant loci and strong candidate genes for growth and fatness traits in two pig populations
}

\author{
Ruimin Qiao ${ }^{\dagger}$, Jun Gao ${ }^{\dagger}$, Zhiyan Zhang, Lin Li, Xianhua Xie, Yin Fan, Leilei Cui, Junwu Ma, Huashui Ai, Jun Ren*
} and Lusheng Huang

\begin{abstract}
Background: Recently, genome-wide association studies (GWAS) have been reported on various pig traits. We performed a GWAS to analyze 22 traits related to growth and fatness on two pig populations: a White Duroc $\times$ Erhualian $F_{2}$ intercross population and a Chinese Sutai half-sib population.

Results: We identified 14 and 39 loci that displayed significant associations with growth and fatness traits at the genome-wide level and chromosome-wide level, respectively. The strongest association was between a $750 \mathrm{~kb}$ region on SSC7 (SSC for Sus scrofa) and backfat thickness at the first rib. This region had pleiotropic effects on both fatness and growth traits in $\mathrm{F}_{2}$ animals and contained a promising candidate gene HMGA1 (high mobility group AT-hook 1). Unexpectedly, population genetic analysis revealed that the allele at this locus that reduces fatness and increases growth is derived from Chinese indigenous pigs and segregates in multiple Chinese breeds. The second strongest association was between the region around $82.85 \mathrm{Mb}$ on SSC4 and average backfat thickness. PLAG1 (pleiomorphic adenoma gene 1), a gene under strong selection in European domestic pigs, is proximal to the top SNP and stands out as a strong candidate gene. On SSC2, a locus that significantly affects fatness traits mapped to the region around the IGF2 (insulin-like growth factor 2) gene but its non-imprinting inheritance excluded IGF2 as a candidate gene. A significant locus was also detected within a recombination cold spot that spans more than $30 \mathrm{Mb}$ on SSCX, which hampered the identification of plausible candidate genes. Notably, no genome-wide significant locus was shared by the two experimental populations; different loci were observed that had both constant and time-specific effects on growth traits at different stages, which illustrates the complex genetic architecture of these traits.
\end{abstract}

Conclusions: We confirm several previously reported QTL and provide a list of novel loci for porcine growth and fatness traits in two experimental populations with Chinese Taihu and Western pigs as common founders. We showed that distinct loci exist for these traits in the two populations and identified HMGA1 and PLAG1 as strong candidate genes on SSC7 and SSC4, respectively.

\section{Background}

Domestic pigs display great phenotypic diversity, that is attributable to approximately 10000 years of natural and artificial selection [1]. Currently, Western commercial pigs show divergent phenotypes compared to Chinese indigenous pigs. Western commercial breeds, such as

\footnotetext{
* Correspondence: renjunjxau@hotmail.com

${ }^{\dagger}$ Equal contributors

Key Laboratory for Animal Biotechnology of Jiangxi Province and the Ministry of Agriculture of China, Jiangxi Agricultural University, Nanchang,
} China

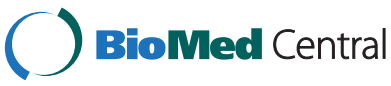

Large White, Landrace, Duroc and Pietrain, have experienced intensive selection for lean pork production in the past decades. Their excellent performance, including fast growth and a high lean percentage, has led them to dominate the global pig industry. Conversely, Chinese indigenous breeds have been historically selected for fat deposition, since fat was an important source of energy for Chinese farmers in ancient times. These breeds are characterized by obesity and a slow growth rate but good meat quality and excellent adaptability to diverse environments. In general, Chinese 
native pigs have average daily gains of $\sim 400 \mathrm{~g} / \mathrm{d}$ and a lean percentage of less than $45 \%$, which are very different from the average daily gain of more than $800 \mathrm{~g} / \mathrm{d}$ and a lean percentage greater than $60 \%$ in Western commercial pigs [2].

To dissect the molecular basis of the divergent phenotypes seen between Chinese and Western pigs, researchers have established multiple $\mathrm{F}_{2}$ intercross populations using Chinese and Western breeds as founder animals [3-6]. Genome scans have been performed on these experimental populations using sparse microsatellite markers across the pig genome to identify quantitative trait loci (QTL) for a variety of traits. For growth and fatness traits, a total of 2623 QTL have been deposited to date in the pig QTL database (http://www.animalgenome.org/ cgi-bin/QTLdb/SS/index). Notably, QTL that are significantly associated with growth and fatness have been consistently identified on pig chromosomes (SSC for Sus scrofa chromosome) 1, 2, 4, 6, 7 and $\mathrm{X}$ using $\mathrm{F}_{2}$ intercross populations of Chinese and Western origin [3-6]. These findings have advanced our understanding of the genetic architecture of porcine growth and fatness traits. Nevertheless, the resolution of traditional QTL mapping is relatively poor due to the limited number of recombination events in the $\mathrm{F}_{2}$ crosses; confidence intervals are generally on the order of $\sim 20 \mathrm{cM}$. Such large regions can contain an abundance of genes, which hampers the prioritization of plausible candidate genes. Thus, the causal variants that underlie the identified QTL remain poorly understood. To date, only one nucleotide, in intron 3 of the IGF2 (insulin-like growth factor 2) gene on SSC2, has been convincingly shown to cause a major QTL effect on muscle mass and heart weight [7].

Recently, genomic tools have revolutionized genetic studies of complex traits. Large human or animal populations can be affordably genotyped using high-density SNP (single nucleotide polymorphism) arrays. The resulting data, together with advanced statistical tools, allow researchers to perform genome-wide mapping of loci for phenotypic traits with greater power and higher accuracy than with traditional QTL mapping. The socalled genome-wide association study (GWAS) mapping approach has been successfully implemented in a growing list of species, including pigs [8-12]. Here, we report the results of GWAS mapping of loci for porcine growth and fatness traits using two experimental populations: a White Duroc $\times$ Erhualian $\mathrm{F}_{2} / \mathrm{F}_{3}$ intercross population and a Chinese Sutai half-sib population. The findings confirm our previous QTL mapping results and, more importantly, reveal a list of novel loci, refine the confidence intervals, and identify strong candidate genes for the major and multifaceted QTL on SSC4 and SSC7.

\section{Methods}

\section{Ethics statement}

All procedures involving animals were in compliance with the guidelines for the care and use of experimental animals established by the Ministry of Agriculture of China. The ethics committee of Jiangxi Agricultural University specifically approved this study.

\section{Animals and phenotypes}

Two experimental populations were used in this study: a White Duroc $\times$ Erhualian $\mathrm{F}_{2} / \mathrm{F}_{3}$ intercross population and a Chinese Sutai half-sib population. The White Duroc $\times$ Erhualian intercross population was established as previously described [13]. Briefly, two White Duroc boars were mated to 17 Erhualian sows. From this first cross, nine $F_{1}$ boars and $59 \mathrm{~F}_{1}$ sows were then intercrossed to produce a total of $1912 \mathrm{~F}_{2}$ animals in six batches and $560 \mathrm{~F}_{3}$ animals in two batches. Sutai pigs are a Chinese synthetic breed that is derived from Chinese Taihu (50\%) and Western Duroc (50\%) following more than 18 generations of artificial selection. All piglets were weaned at day 46 and males were castrated at day 90. All fattening pigs were raised under controlled indoor conditions in the experimental farm of Jiangxi Agricultural University (China) and were fed ad libitum on a diet containing 16\% crude protein, $3100 \mathrm{~kJ}$ digestible energy and $0.78 \%$ lysine. Pigs were slaughtered for phenotype recording at the age of $240 \pm 3$ days. Body weight was measured at birth and at days $21,46,120,210$ and 240 for each $F_{2}$ and $F_{3}$ individual. For Sutai pigs, body weight was also recorded at these growth stages except at day 46. Average daily weight gain at multiple time intervals was calculated. After slaughter, seven fatness-related traits, including backfat thickness at the shoulder, the first rib, the last rib and the hip, and weight of leaf fat, veil fat and abdominal fat, were recorded for all individuals from both populations. A total of $930 \mathrm{~F}_{2}$ individuals and 432 Sutai pigs from five sires and 60 dams were used for GWAS mapping in this study.

\section{Genotypes and quality control}

Genomic DNA of each animal was extracted from ear or tail tissue using a standard phenol/chloroform method. A total of 1017 animals, including $930 \mathrm{~F}_{2}$ pigs, 87 parental pigs in the $F_{2}$ cross and 432 Sutai pigs, were genotyped for 62163 SNPs using the Illumina PorcineSNP60 BeadChip according to the manufacturer's protocol. Quality control procedures were carried out using Plink v 1.07 [14], and the same quality control criteria were applied to the SNP data from the two populations. Briefly, animals with a call rate greater than 0.9 and mendelian error rate smaller than 0.05, and SNPs with a call rate greater than 0.9 , minor allele frequency higher than $0.05, P$ value greater than $10^{-6}$ for the Hardy-Weinberg equilibrium test and mendelian error rate smaller than 0.1 were included. 
A final set of 39788 informative SNPs from 1017 animals from the $F_{2}$ cross and the 432 Sutai pigs were used for subsequent analyses.

\section{Single-marker GWAS}

The allelic effect of each SNP on phenotypic traits was tested using a general linear mixed model [15-17]. The model included a random polygenic effect, and the variance-covariance matrix was proportional to genomewide identity by state [18]. The formula of the model was $\mathbf{Y}=\mathbf{X b}+\mathbf{S a}+\mathbf{Z u}+\mathbf{e}$, where $\mathbf{Y}$ is the vector of phenotypes; b is the estimator of fixed effects including sex, batch and carcass weight; a is the SNP substitution effect; and $\mathbf{u}$ is the vector of random additive genetic effects following the multinormal distribution $\mathbf{u} \sim \mathrm{N}\left(0, \mathbf{G} \sigma_{\alpha}{ }^{2}\right)$, in which $\mathbf{G}$ is the genomic relationship matrix that was constructed based on SNPs, as described in [19], and $\sigma_{\alpha}{ }^{2}$ is the polygenetic additive variance. $\mathbf{X}$ and $\mathbf{Z}$ are the incidence matrices for $\mathbf{b}$ and $\mathbf{u}, \mathbf{S}$ is the incidence vector for a, and $\mathbf{e}$ is a vector of residual errors with a distribution of $\mathrm{N}\left(0, \mathbf{I} \sigma_{\mathrm{e}}{ }^{2}\right)$. All single-marker GWAS analyses were conducted using the GenABEL package [20,21]. Based on the Bonferroni method, the genome-wide significance threshold was defined as $0.05 / \mathrm{N}$, where $\mathrm{N}$ is the number of informative SNPs. The chromosome-wide significance threshold was defined as $0.1 / \mathrm{N}$.

\section{Linkage disequilibrium and linkage association (LDLA) analysis}

First, the haplotype of each chromosome was reconstructed for each animal in the $F_{2}$ population using the 60 K SNP data, pedigree information and a Hidden Markov model [22]. The model simultaneously phased SNP genotypes and assigned the ensuing haplotypes to a predetermined number of ancestral haplotypes that was set at 20 in this study. Then, for each locus, the effect of these ancestral haplotypes was estimated using a mixed model framework: $\mathbf{Y}=\mathbf{X b}+\mathbf{Z u}+\mathbf{e}$ [23], where $\mathbf{Y}$ is the vector of phenotypes; $\mathbf{b}$ is the estimator of fixed effects including sex, batch and carcass weight; $\mathbf{X}$ and $\mathbf{Z}$ are the incidence matrices for $\mathbf{b}$ and $\mathbf{u} ; \mathbf{u}$ is the random additive genetic effect following the multinormal distribution $\mathbf{u} \sim \mathrm{N}\left(0, \mathbf{G} \sigma_{\alpha}{ }^{2}\right)$, in which $\mathbf{G}$ is the individual-individual similarities matrix which was calculated from wholegenome information and $\sigma_{\alpha}{ }^{2}$ is the polygenetic additive variance; and $\mathbf{e}$ is a vector of residual errors with a distribution of $\mathrm{N}\left(0, \mathbf{I} \sigma_{\mathrm{e}}{ }^{2}\right)$. The ancestral haplotype-based LDLA analysis can use both within-family linkage information and across-family linkage disequilibrium information resulting from historical recombination events in ancestors of founder animals in the $F_{2}$ population. We conducted the LDLA analysis using $\mathrm{R}$ scripts that were written for this purpose. The $95 \%$ confidence interval
(CI) was determined by a LOD score drop-off of 2 from the value of the most significant loci.

\section{Haplotype analysis at the major loci on SSC7}

For the major locus on SSC7, we constructed haplotypes for all 17 founder sows and two founder boars using the DualPHASE software [24]. The effects of the reconstructed haplotypes on phenotypic traits were evaluated using marker-assisted segregation analysis and multiple comparison tests, as described previously [7]. The putative critical region on SSC7 was identified by haplotypesharing and LDLA-mapping analyses. Furthermore, haplotypes that corresponded to the refined critical region at the SSC7 locus were reconstructed for 589 individuals from 31 Chinese and Western breeds using $60 \mathrm{~K}$ SNPs from our previous study [25] and fastPHASE software [26]. Haplotypes with frequencies greater than 0.05 were used to construct a neighbor-joining tree in MEGA 5.0 with 1000 bootstrap iterations [27].

\section{Results}

Phenotypic values

Table S1 (see Additional file 1: Table S1) presents the phenotypic values of 14 growth traits and eight fatness traits measured in the White Duroc $\times$ Erhualian $F_{2}$ population and Chinese Sutai pigs. Both populations showed comparable body weights at early stages, while the Sutai pigs had obviously lower body weights at adult ages (days 210 and 240). For fatness traits, Sutai pigs exhibited leaner phenotypes than the $F_{2}$ animals; indeed, all fatness-related measurements were greater $(P<0.05)$ in $\mathrm{F}_{2}$ animals.

\section{Summary of GWAS results}

In total, we identified 14 loci on seven chromosomes that exceeded the genome-wide significance thresholds; these included two loci on SSC2 and two on SSC3, four on SSC4, three on SSC7 and one each on SSC10, 14 and X. These prominent loci were associated with three growth traits and eight fatness traits (Table 1). However, none of them was shared between the two experimental populations. In addition, we detected 39 loci that demonstrated significance at the chromosome significant level (referred to as suggestive loci); these loci were located on all autosomes except SSC13, 16 and 17 (see Additional file 2: Table S2). Again, the $\mathrm{F}_{2}$ and Sutai populations showed distinct association signals at most of these loci. In Sutai pigs, no locus displayed a significant association to any fatness trait at the whole-genome level (see Additional file 3: Figure S1).

\section{Genome-wide significant loci and strong candidate genes in the $F_{2}$ population \\ SSC7}

Four major loci that affect fat deposition and growth were identified on SSC2, 4, 7 and $\mathrm{X}$ in the $\mathrm{F}_{2}$ population, 
Table 1 QTL of genome-wide significance identified by GWAS for growth and fatness traits in White Duroc $\times$ Erhualian $F_{2}$ pigs and Sutai pigs

\begin{tabular}{|c|c|c|c|c|c|c|c|c|c|c|c|c|}
\hline \multirow[t]{2}{*}{$\mathrm{Chr}^{1}$} & \multirow[t]{2}{*}{ Trait } & \multirow[t]{2}{*}{ Abbreviation } & \multirow[t]{2}{*}{ Pop $^{2}$} & \multirow[t]{2}{*}{$\mathrm{N}_{\mathrm{snp}}{ }^{3}$} & \multirow[t]{2}{*}{ Top SNP } & \multirow[t]{2}{*}{ Position (bp) } & \multirow[t]{2}{*}{$\mathrm{MAF}^{4}$} & \multicolumn{2}{|c|}{ Frequency $^{5}$} & \multirow[t]{2}{*}{ Effect } & \multirow[t]{2}{*}{$P$-value } & \multirow{2}{*}{$\begin{array}{l}\text { Candidate } \\
\text { gene }\end{array}$} \\
\hline & & & & & & & & Duroc & Erhualian & & & \\
\hline \multirow[t]{2}{*}{2} & $\begin{array}{l}\text { Body weight at day } \\
21\end{array}$ & BW21 & Sutai & 1 & ss107909052 & 101694375 & 0.05 & NA & NA & 0.67 & 2.66E-08 & ARRDC3 \\
\hline & $\begin{array}{l}\text { Backfat thickness at } \\
\text { the hip }\end{array}$ & HBFT & $\mathrm{F}_{2}$ & 4 & ss131060419 & 920370 & 0.28 & 0.50 & 1.00 & -0.25 & $2.59 \mathrm{E}-07$ & \\
\hline \multirow[t]{2}{*}{3} & $\begin{array}{l}\text { Body weight at day } \\
21\end{array}$ & BW21 & Sutai & 4 & ss131231938 & 130852806 & 0.06 & NA & NA & 1.05 & $6.70 \mathrm{E}-08$ & \\
\hline & $\begin{array}{l}\text { Average daily gain } \\
\text { from day } 0 \text { to } 21\end{array}$ & ADG0-21 & Sutai & 2 & ss131231938 & 130852806 & 0.06 & NA & NA & 0.06 & $1.22 \mathrm{E}-08$ & \\
\hline \multirow[t]{7}{*}{4} & $\begin{array}{l}\text { Average backfat } \\
\text { thickness }\end{array}$ & ABFT & $F_{2}$ & 8 & ss131269801 & 82850635 & 0.46 & 1.00 & 0.00 & -0.28 & 8.50E-09 & PLAG1 \\
\hline & $\begin{array}{l}\text { Backfat thickness at } \\
\text { the first rib }\end{array}$ & FRBFT & $F_{2}$ & 4 & ss131269801 & 82850635 & 0.46 & 1.00 & 0.00 & -0.31 & $6.04 \mathrm{E}-08$ & \\
\hline & Leaf fat weight & LFW & $\mathrm{F}_{2}$ & 6 & ss131269801 & 82850635 & 0.46 & 1.00 & 0.00 & -0.31 & 3.80E-08 & \\
\hline & $\begin{array}{l}\text { Backfat thickness at } \\
\text { the hip }\end{array}$ & HBFT & $\mathrm{F}_{2}$ & 4 & ss131270942 & 88978451 & 0.44 & 1.00 & 0.00 & -0.32 & 1.46E-08 & \\
\hline & $\begin{array}{l}\text { Body weight at day } \\
21\end{array}$ & BW21 & Sutai & 4 & ss131247062 & 134935929 & 0.05 & NA & NA & 1.09 & 1.35E-07 & \\
\hline & $\begin{array}{l}\text { Average daily gain } \\
\text { from day } 0 \text { to } 21\end{array}$ & ADG0-21 & Sutai & 2 & ss131247062 & 134935929 & 0.05 & NA & NA & 0.06 & $1.48 \mathrm{E}-08$ & \\
\hline & $\begin{array}{l}\text { Average daily gain } \\
\text { from day } 0 \text { to } 21\end{array}$ & ADG0-21 & Sutai & 2 & ss107817770 & 133079451 & 0.08 & NA & NA & 0.06 & $1.48 \mathrm{E}-08$ & \\
\hline \multirow[t]{9}{*}{7} & $\begin{array}{l}\text { Average backfat } \\
\text { thickness }\end{array}$ & ABFT & $F_{2}$ & 167 & ss107837325 & 34803564 & 0.46 & 0.00 & 0.94 & -0.62 & $7.63 E-32$ & HMGA1 \\
\hline & $\begin{array}{l}\text { Backfat thickness at } \\
\text { the first rib }\end{array}$ & FRBFT & $\mathrm{F}_{2}$ & 184 & ss107837325 & 34803564 & 0.46 & 0.00 & 0.94 & -0.7 & 3.39E-32 & \\
\hline & Leaf fat weight & LFW & $F_{2}$ & 165 & ss107837325 & 34803564 & 0.46 & 0.00 & 0.94 & -0.66 & $2.61 \mathrm{E}-30$ & \\
\hline & $\begin{array}{l}\text { Backfat thickness at } \\
\text { the hip }\end{array}$ & HBFT & $\mathrm{F}_{2}$ & 155 & ss107837325 & 34803564 & 0.46 & 0.00 & 0.94 & -0.78 & $6.61 \mathrm{E}-28$ & \\
\hline & $\begin{array}{l}\text { Backfat thickness at } \\
\text { the last rib }\end{array}$ & LRBFT & $\mathrm{F}_{2}$ & 166 & ss107837325 & 34803564 & 0.46 & 0.00 & 0.94 & -0.57 & $2.17 E-24$ & \\
\hline & $\begin{array}{l}\text { Backfat thickness at } \\
\text { the shoulder }\end{array}$ & SBFT & $F_{2}$ & 115 & ss107837325 & 34803564 & 0.46 & 0.00 & 0.94 & -0.49 & $1.01 \mathrm{E}-17$ & \\
\hline & $\begin{array}{l}\text { Abdominal fat } \\
\text { weight }\end{array}$ & AFW & $\mathrm{F}_{2}$ & 121 & ss107837325 & 34803564 & 0.46 & 0.00 & 0.94 & -0.15 & $1.79 \mathrm{E}-14$ & \\
\hline & Veil fat weight & VFW & $F_{2}$ & 29 & ss107806758 & 35177641 & 0.45 & 0.00 & 0.88 & -0.14 & 4.92E-08 & \\
\hline & $\begin{array}{l}\text { Body weight at day } \\
240\end{array}$ & BW210 & $\mathrm{F}_{2}$ & 3 & ss131342496 & 32957768 & 0.49 & 0.00 & 1.00 & -5.44 & $6.77 \mathrm{E}-07$ & \\
\hline 10 & $\begin{array}{l}\text { Average daily gain } \\
\text { from day } 0 \text { to } 21\end{array}$ & ADG0-21 & Sutai & 1 & ss478939281 & 13838740 & 0.08 & NA & NA & 0.02 & 8.89E-07 & \\
\hline 14 & $\begin{array}{l}\text { Average daily gain } \\
\text { from day } 0 \text { to } 21\end{array}$ & ADG0-21 & Sutai & 1 & ss131505908 & 151638018 & 0.09 & NA & NA & 0.05 & 4.46E-08 & \\
\hline$x$ & $\begin{array}{l}\text { Backfat thickness at } \\
\text { the hip }\end{array}$ & HBFT & $F_{2}$ & 12 & ss131561996 & 62086511 & 0.21 & 0.50 & 0.00 & -0.31 & $6.84 \mathrm{E}-12$ & \\
\hline
\end{tabular}

${ }^{1}$ Chromosome; ${ }^{2}$ population; ${ }^{3}$ number of SNPs that surpass the genome-wide significance level; ${ }^{4}$ minor allele frequency; ${ }^{5}$ allele frequencies of top SNPs in two Duroc founder boars and 17 Erhualian founder sows from the $F_{2}$ intercross resource population; NA, not available for Sutai pigs.

which confirmed our previously published QTL mapping results [28]. Of these, the most striking locus was located at about 34 to $36 \mathrm{Mb}$ on SSC7 (referred to hereafter as the SSC7 locus; Table 1) and was significantly associated with all fatness traits (Figure 1) and body weight at day 240 (Figure 2). The top SNP for the associated traits was ss107837325 at 34803564 bp on this chromosome. At this location, the allele substitution effect accounted for more than $5 \mathrm{~mm}$ of backfat thickness and $5.44 \mathrm{~kg}$ of body weight at day 240 (Table 1), explaining 40\% of 


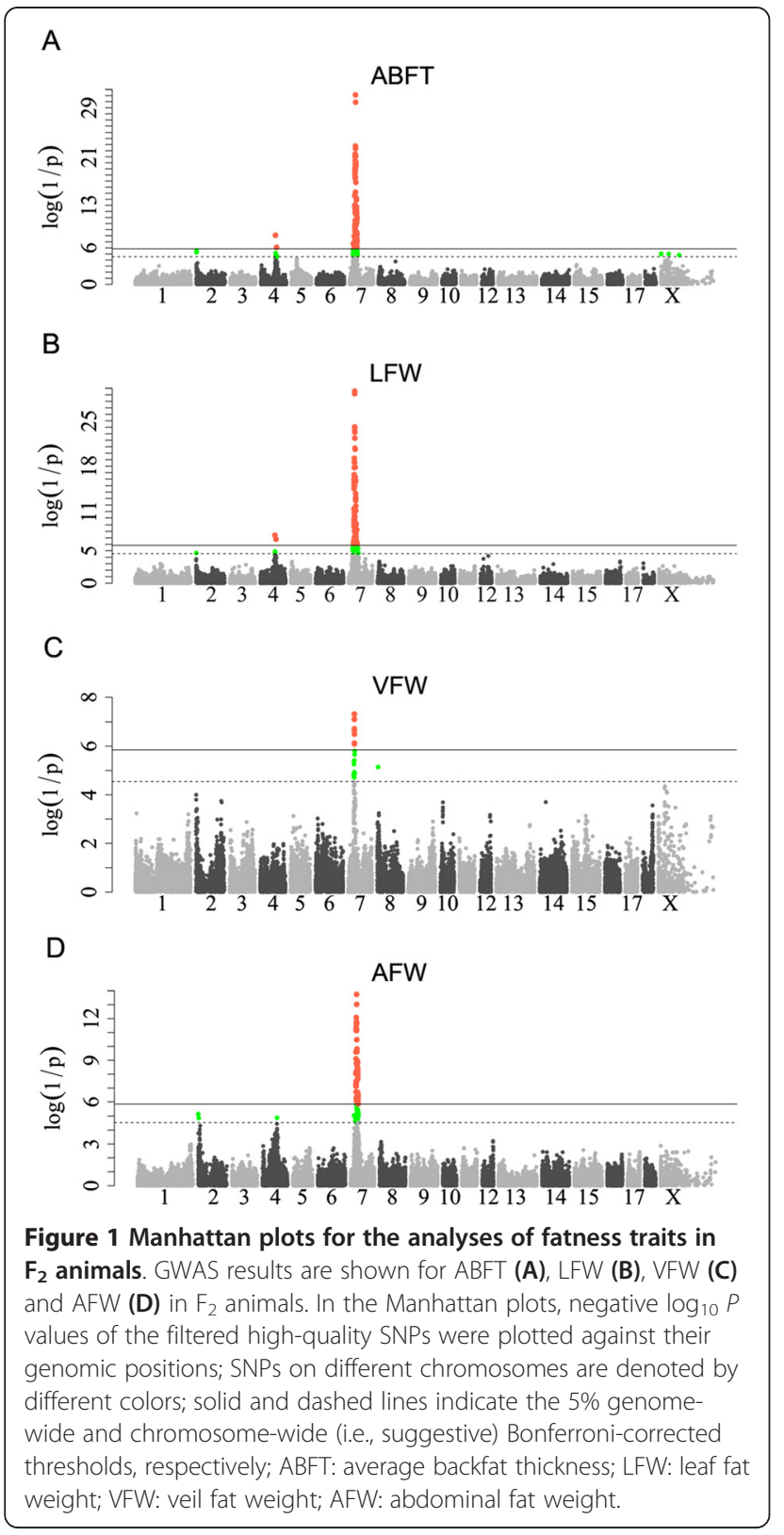

phenotypic variance in these traits. By applying the LDLA and LOD-drop-off-2 approaches, we defined the 95\% CI of the SSC7 locus as a 2.1-Mb region that was flanked by ss131342658 (33 $299125 \mathrm{bp}$ ) and ss131344365 (35 422 882 bp) (Figure 3A). Next, we conducted a haplotype analysis of a $\sim 3.2-\mathrm{Mb}$ region (between 33299125 and 36 $475968 \mathrm{bp}$ ) surrounding the $95 \% \mathrm{CI}$ of the SSC7 locus, using 17 founder sows and two founder boars from the $\mathrm{F}_{2}$ population. We showed that a $\sim 1.7-\mathrm{Mb}$ segment between 34673190 (ss131343640) and 36329680 bp (ss478941605) was shared by 30 Erhualian founder chromosomes (Figure 3B). We then reconstructed haplotypes corresponding to this $1.7-\mathrm{Mb}$ segment in all $\mathrm{F}_{2}$ animals and evaluated the effects of these haplotypes on average backfat thickness (ABFT) and leaf fat weight (LFW) in the $\mathrm{F}_{2}$ animals. The shared haplotype appeared to be a haplotype that was significantly associated with decreased fat deposition (referred to hereafter as Q haplotype) (see Additional file 4: Figure S2). This result was in accordance with previous reports [3,4,29-31], but was also unexpected considering that the Erhualian is an obese Chinese pig breed.

We noted that the haplotypes that were associated with increased fat deposition (referred to hereafter as $\mathrm{q}$ haplotypes) were derived from both White Duroc founder boars (q1 and q2, Figure 3B) and Erhualian founder sows (q3, Figure 3B). One haplotype (referred to as q?), originating from Erhualian founders 142 and 146, seemed to be a q-type haplotype (Figure 3B). However, the QTL status of this haplotype could not be deduced, since it was carried by only 10 of the $930 \mathrm{~F}_{2}$ animals that we had genotyped using the $60 \mathrm{~K} \mathrm{SNP}$ panel (see Additional file 4: Figure S2A). To determine if this haplotype was a $\mathrm{Q}$ or a $q$ haplotype, we further examined the White Duroc $x$ Erhualian intercross population. In our previous study [32], we had genotyped all $F_{2}$ and $F_{3}$ descendants of individuals 142 and 146, for a total of 20 markers around the SSC7 QTL region. When we examined this marker and pedigree information, we found one additional $F_{2}$ animal (2045) and six $\mathrm{F}_{3}$ animals that had inherited the q? haplotype from individuals 142 and 146 (see Additional file 5: Figure S3). We then conducted further statistical analyses on the phenotypic data of the 17 animals. We found that individuals carrying the $\mathrm{q}$ ? haplotype had obviously higher ABFT and LFW than those with the Q haplotype (see Additional file 4: Figure S2B). We thus assumed that the q? haplotype is a q-type haplotype. To obtain further evidence for this assumption, we compared the phenotypic differences in ABFT and LFW between q?, q-, and Q-type haplotype pairs (see Additional file 6: Table S3). The pairwise $t$-test statistics indicated that there was no significant difference in $\operatorname{ABFT}(P=0.10)$ and LFW $(P=0.28)$ between qq and qq? individuals. Additionally, there was no significant difference in ABFT $(P=0.29)$ and LFW $(P=0.29)$ between $\mathrm{Qq}$ and $\mathrm{Qq}$ ? individuals. In comparison, $\mathrm{QQ}$ animals had significantly lower ABFT $(P=0.05)$ and LFW $(P=0.06)$ than Qq? animals. Although we did not observe significant differences in $\operatorname{ABFT}(P=0.15)$ and LFW ( $P=0.17$ ) between Qq and qq? animals, Qq individuals tended to have lower ABFT $(2.95 \pm 0.77$ vs. $3.20 \pm$ $0.41 \mathrm{~cm})$ and LFW $(2086.6 \pm 1076.2$ vs. $2416.3 \pm 864 . \mathrm{g})$ than qq? individuals. Altogether, our findings indicate that the $\mathrm{q}$ ? haplotype is most likely a q haplotype that increases fat deposition, rather than a $Q$ haplotype that decreases fat deposition. All the q-type haplotypes were distinct from the Q-type haplotypes in the 1.7-Mb Q-sharing segment (Figure 3C). 


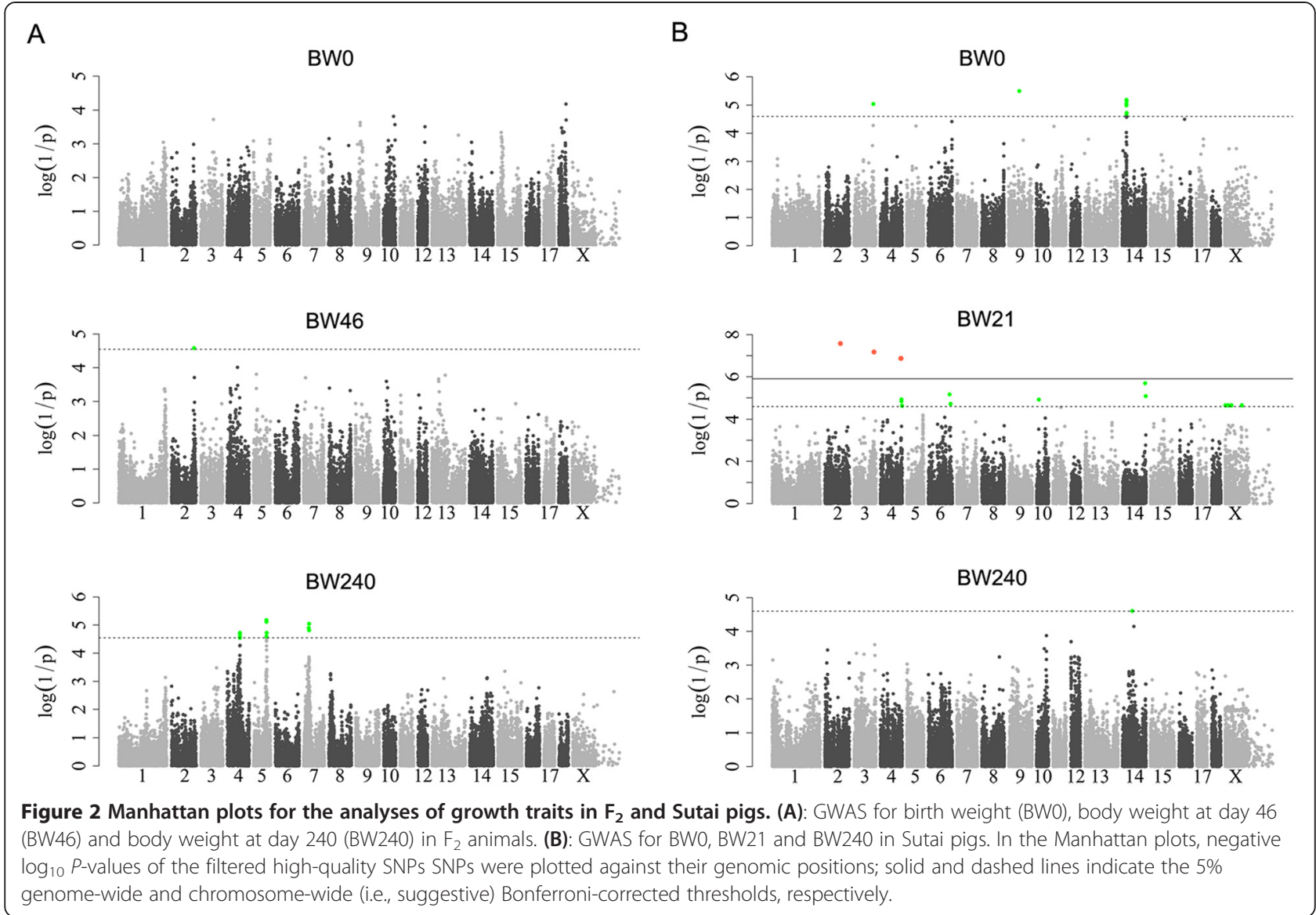

We were particularly interested in a $\sim 750$-kb region on SSC7 (between 34 673190 and $35422882 \mathrm{bp}$ ) that was contained within both the 2.1-Mb 95\% CI and the 1.7-Mb Q-sharing segment (Figure $3 \mathrm{C}$ ). It is most likely that this region encompasses the gene responsible for the SSC7 locus. To investigate the population genetics and evolutionary history at this SSC7 locus, we reconstructed haplotypes corresponding to the critical 750-kb region for 589 individuals from 31 diverse Chinese and Western breeds (Table 2), which had been genotyped for 62163 SNPs using the Illumina Porcine $60 \mathrm{~K}$ DNA chip [25]. A total of 27 haplotypes with frequencies greater than 0.05 were subsequently used to construct a neighborjoining (NJ) tree. The phylogenetic tree clearly illustrated that the Q-type haplotype is of Chinese origin (Figure 4). Of the 27 haplotypes, 14 occurred exclusively in Chinese indigenous pigs, and two were predominately $(>0.8)$ present in Western pigs. Notably, the Q-type haplotype that we identified in the $F_{2}$ population was present in multiple Chinese indigenous breeds, many of which carry the $\mathrm{Q}$ allele at considerably high frequencies (Table 2). Conversely, this haplotype was nearly absent in Western breeds and Chinese wild boars, which suggests that the causal variants underlying the SSC7 QTL originated after the domestication of Chinese wild boars. Further investigations in multiple Chinese breeds of the minimal shared haplotype that carries the Q allele would be useful to identify the causative mutation underlying the SSC7 locus. Given that a number of Chinese breeds segregate for this major locus, characterization of the causal variant would greatly contribute to the genetic improvement of growth and fatness traits in Chinese indigenous pigs.

The 750-kb critical region on SSC7 harbors eight annotated genes, including GRM4 (glutamate receptor, metabotropic 4), HMGA1 (high mobility group AT-hook 1), C6ORF1 (chromosome 6 open reading frame 1), NUDT3 (nudix (nucleoside diphosphate linked moiety X)-type motif 3), RSP10 (ribosomal protein S10), SPDEF (SAM pointed domain containing ETS transcription factor), PACSIN1 (protein kinase $C$ and casein kinase substrate in neurons 1) and C6ORF106 (chromosome 6 open reading frame 106) (Figure 3C). Of these eight genes, NUDT3 could be a candidate gene for the SSC7 locus, as NUDT3 variants have been associated with changes in human body mass index values [33] and height [34]. However, HMGA1 is another promising candidate gene since it is functionally related to growth and fat metabolism. It encodes a non-histone protein that plays a role in multiple 


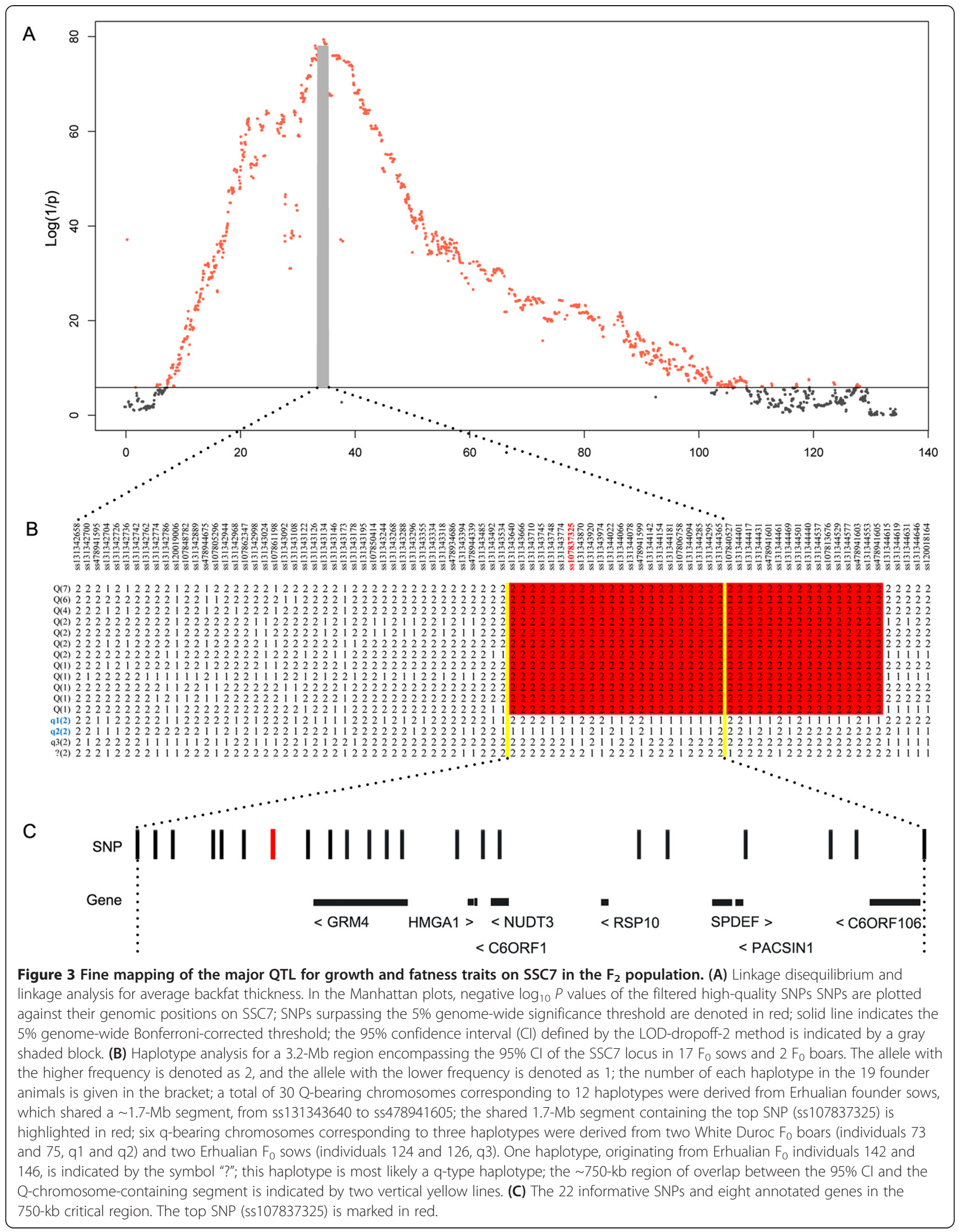


Table 2 Frequency of the Q-bearing haplotype at the SSC7 locus in 31 Chinese and Western pig breeds

\begin{tabular}{|c|c|c|c|}
\hline Breed & Origin & Number & Frequency \\
\hline \multicolumn{4}{|l|}{ Indigenous Chinese breeds } \\
\hline Erhualian & Jiangsu & 32 & 0.83 \\
\hline Dongshan & Guangxi & 15 & 0.73 \\
\hline Min & Heilongjiang & 22 & 0.66 \\
\hline Hetao Large Ear & Inner Mongolia & 16 & 0.59 \\
\hline Tongcheng & Hubei & 16 & 0.59 \\
\hline Rongchang & Chongqing & 18 & 0.58 \\
\hline Neijiang & Sichuan & 16 & 0.56 \\
\hline Shaziling & Hunan & 11 & 0.55 \\
\hline Kele & Guizhou & 10 & 0.55 \\
\hline Guangdong Dahuabai & Guangdong & 16 & 0.53 \\
\hline Luchuan & Guangxi & 18 & 0.47 \\
\hline Ganxi & Jiangxi & 13 & 0.46 \\
\hline Tibetan (Diqing) & Yunnan & 19 & 0.34 \\
\hline Bamaxiang & Guangxi & 16 & 0.34 \\
\hline Mingguang Small Ear & Yunnan & 16 & 0.34 \\
\hline Bamei & Qinghai & 16 & 0.31 \\
\hline Tibetan (Milin) & Tibet & 16 & 0.25 \\
\hline Wuzhishan & Hainan & 16 & 0.25 \\
\hline Diannan Small-ear & Yunnan & 15 & 0.10 \\
\hline Congjiangxiang & Guizhou & 16 & 0.09 \\
\hline Tibetan (Litang) & Sichuan & 16 & 0.06 \\
\hline Tibetan (Hezuo) & Gansu & 21 & 0.05 \\
\hline Tibetan (Gongbujiangda) & Tibet & 29 & 0.03 \\
\hline Laiwu & Shandong & 18 & 0.03 \\
\hline Wild boar & Jiangxi & 20 & 0.00 \\
\hline Jinhua & Zhejiang & 13 & 0.00 \\
\hline Licha Black & Shandong & 14 & 0.00 \\
\hline \multicolumn{4}{|l|}{ Chinese synthetic breed } \\
\hline Sutai & Jiangsu & 15 & 0.03 \\
\hline \multicolumn{4}{|c|}{ Western commercial breeds } \\
\hline Large White & France & 35 & 0.01 \\
\hline Landrace & Denmark & 35 & 0.00 \\
\hline Duroc & U.S.A & 40 & 0.00 \\
\hline
\end{tabular}

cellular processes. HMGA1 can serve as a modulator of IGF1 (insulin-like growth factor 1) activity and consequently regulates glucose uptake [35]. It is also significantly associated with human height [36]. Moreover, a STRING protein-protein interaction network shows that HMGA1 can bind with PPARG (peroxisome proliferatoractivated receptor gamma), a key regulator of fat-cell differentiation and glucose homeostasis [37] (see Additional file 7: Figure S4). In our previous study, we genotyped one HMGA1 variant $(\mathrm{g} .3135 \mathrm{C}>\mathrm{T})$ in all individuals of the $\mathrm{F}_{2}$ population [32]. When we included this HMGA1 SNP in the single-marker GWAS, it was the most significant SNP for backfat thickness on SSC7 (see Additional file 8: Figure S5) and exhibited the same strength of association as the top SNP of the original GWAS (ss107837325). This finding further supports $H M G A 1$ as a highly plausible candidate gene responsible for the SSC7 locus. It would thus be worthwhile to further investigate functional variants in HMGA1 that underlie the QTL effect on SSC7.

The 750-kb interval is approximately $700 \mathrm{~kb}$ upstream of the PPARD gene (between 36141606 and 36215 $260 \mathrm{bp}$ ), which has been identified as the gene responsible for ear size in the $F_{2}$ population [32]. Here, we show that PPARD falls outside the 95\% CI of the SSC7 locus, which indicates that the growth and fatness traits associated with this locus are not affected by polymorphisms in the PPARD gene. When we included PPARD G32E SNP, the causal mutation for ear size, in the GWAS analysis of fatness traits, we found that this SNP had a much lower association significance than the top SNP ss107837325 (see Additional file 8: Figure S5). This finding provided more evidence that PPARD is not the gene responsible for the SSC7 locus that affects growth and fatness traits.

\section{SSC4}

The second strongest effect on the measured traits was observed within an interval of $7 \mathrm{Mb}$ (between 82 and $89 \mathrm{Mb}$ ) on SSC4. After fitting the effect of the SSC7 locus, the strength of the association between this locus and fatness traits in $F_{2}$ animals exceeded the genomewide significance threshold (see Additional file 9: Figure S6). This region also showed various associations with growth traits in $\mathrm{F}_{2}$ animals, although the strength of the association only reached the chromosome-wide significance level (see Additional file 2: Table S2). The top SNP at this locus was ss131269801, at $82850635 \mathrm{bp}$. This locus corresponds to the well-known FAT1 region, which was identified in the first genome scan for pig QTL [38] and subsequently characterized in multiple pig resource populations [4,39-41]. Multiple FABP (fatty acid binding protein) genes, such as FABP4, have been proposed as candidate genes for this locus $[42,43]$. However, the FABP4 gene is located $9 \mathrm{Mb}$ away from the top SNP in this study. When we corrected for the effect of the top SNP, the GWAS signal was entirely absent from SSC4. Thus, FABP genes are probably not the genes of interest here. Instead, just $\sim 240 \mathrm{~kb}$ downstream from the top SNP lies the PLAG1 (pleiomorphic adenoma gene 1) gene, which has been reported to be associated with bovine stature [44] and human height [36]. Moreover, PLAG1 appears to be one of the top genes that was under selection during the domestication of European pigs, and PLAG1 variants have been associated with 


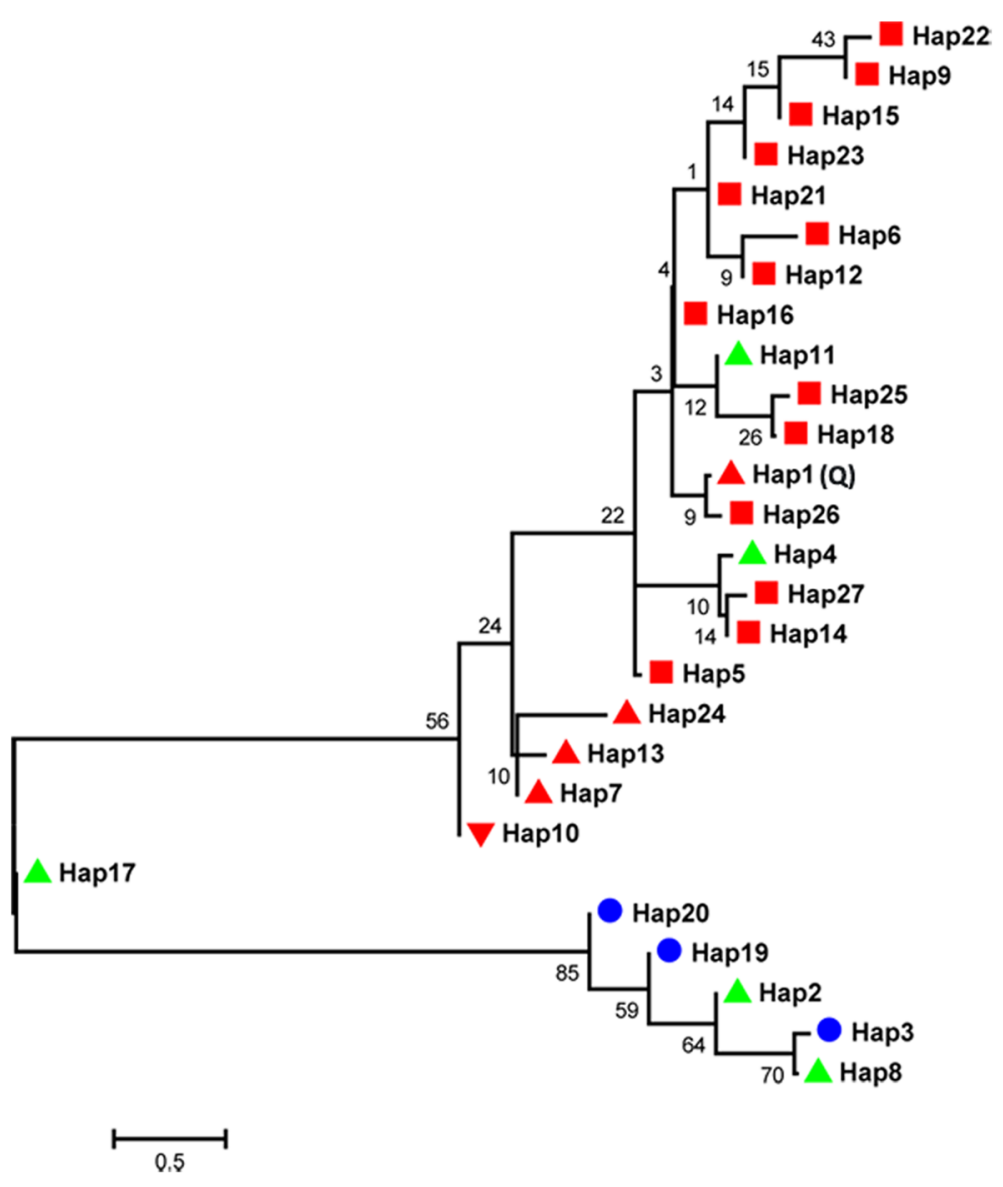

Figure 4 Neighbor-joining tree of 27 major haplotypes for a 750-kb critical region of the major QTL on SSC7. Haplotypes with frequencies greater than 0.05 were used to construct a NJ tree using the 750-kb critical region of SSC7. Hap 1 is the Q-bearing haplotype; haplotypes specific to Chinese indigenous breeds are indicated in red blocks; haplotypes present in both Chinese and Western breeds are highlighted in blue blocks; haplotypes prominently occurring in Chinese breeds and Western breeds are marked in red and green triangular blocks, respectively; haplotypes specific to Chinese indigenous breeds and Chinese synthetic breeds are indicated by red inverted triangular blocks.

growth and fatness traits in a European Wild boar $\times$ Large White $\mathrm{F}_{2}$ intercross population [45]. The PLAG1 region harbors multiple SNPs that show strong signals of selection and marked differences in allele frequency between Asian pigs/wild boars and European domestic pigs [45]. To investigate this, we randomly chose one of the most strongly fixed SNPs in European domestic pigs at $82499373 \mathrm{bp}$ on SSC4, and genotyped this PLAG1 SNP by Sanger sequencing for all animals of the $\mathrm{F}_{2}$ population. The PLAG1 SNP data were then incorporated into the $60 \mathrm{~K} \mathrm{SNP}$ dataset for the singlemarker GWAS, after correction for the effect of the SSC7 locus. Our results showed that the association between the PLAG1 SNP and growth and fatness traits was as strong as that with the top SNP from the $60 \mathrm{~K}$ panel (ss131269801), which was attributable to complete linkage disequilibrium between the two SNPs (data not shown). Based on this, we propose that PLAG1 is a strong candidate gene for the SSC4 locus. It should also be noted that another locus with significant effects on body weight at day 21 in Sutai pigs was identified in a different region on this chromosome, with the top SNP located at 134.935 $929 \mathrm{bp}$.

\section{SSC2}

After correcting for the effects of the SSC7 locus, the effect of SSC2 on fatness traits surpassed the genome-wide significance threshold (see Additional file 9: Figure S6). The finding is consistent with our previous QTL mapping results [28]. The top SNP (ss131060419) was located at the distal end (920 $370 \mathrm{bp}$ ) of SSC2, corresponding to the well-characterized IGF2 gene, which affects muscle mass and fatness in pigs [46]. It is known that IGF2 is a paternally expressed gene. However, the effect at the SSC2 locus disappeared when we applied a paternallyexpressed-imprinting statistical model to the data (data 
not shown). Therefore, IGF2 is likely not the gene responsible for this locus. Another region, around $101.69 \mathrm{Mb}$ on SSC2 had a significant effect on body weight at day 21 in Sutai pigs.

\section{SSCX}

Our previous QTL scans and studies by other researchers have consistently found the presence of a major locus for fatness traits on SSCX based on data from Chinese $\times$ Western intercross populations [5,28,30,47-50] . Here, we confirm these previous findings by identifying a locus of genome-wide significance for backfat thickness at around $62 \mathrm{Mb}$ on SSCX, with the top SNP (ss131561996) at $62086511 \mathrm{bp}$. This region has been shown to be a recombination cold spot that spans $\sim 31 \mathrm{Mb}$ and shows an extremely low rate of recombination in our $\mathrm{F}_{2}$ population $[45,51]$. This low-recombination region harbors a large number of predicted genes [28], which makes it impossible to identify plausible candidate genes.

\section{Loci of genome-wide significance and plausible candidate genes in Sutai pigs}

A total of seven loci of genome-wide significance were identified in Sutai pigs, with one each on SSC2, 10 and 14, and two on SSC3 and two on SSC4 (Table 1). These loci were all associated with growth during the suckling period (from 0 to 21 days) in Sutai pigs but not in $F_{2}$ pigs. The most significant $\left(P=1.22 \times 10^{-8}\right)$ locus was associated with a region on SSC3, but no obvious candidate gene was identified. A locus on SSC2, with its top SNP (ss107909052) at 101694375 bp $\left(P=2.66 \times 10^{-8}\right)$, corresponded to a QTL with chromosome-wide significance for body weight, which had been previously identified in a Chinese Meishan $\times$ Large White population [49]. ARRDC3 (arrestin domain containing 3), $\sim 125 \mathrm{~kb}$ away from this top SNP, appears to be a functionally related candidate gene at this locus. This gene has been reported to play a role in mechanisms that control the regulation of glucose and lipid metabolism and insulin secretion [52,53]. Moreover, the locus on SSC10 that had the strongest signal at $13838740 \mathrm{bp}\left(P=8.89 \times 10^{-7}\right)$, overlaps with a QTL of genome-wide significance that affects body weight at birth in Western hybrid pigs [54]. We did not find any apparent candidate gene in the region around the top SNP (ss478939281) at that locus. The QTL on SSC4 and SSC14 are, to our knowledge, detected for the first time in this study. There were no obvious candidate genes in the vicinity of the top SNPs (ss131247062, ss107817770 and ss131505908) at these two QTL.

\section{Suggestive QTL for growth and fatness traits}

In addition to the significant loci mentioned above, we identified 39 QTL for which a chromosome-wide, but not a genome-wide, association was detected with 20 growth and fatness traits (see Additional file 2: Table S2). These QTL included two each on SSC1, 3, 4, 9 and 15; seven on SSC2; three each on SSC5, 6, 7, and 8; one each on SSC10, 11, 12, 18 and X; and five on SSC14. However, most of these QTL were not shared between the $\mathrm{F}_{2}$ and Sutai populations. The suggestive QTL on SSC2, 4 and 7 overlapped perfectly with the abovedescribed prominent QTL in the $F_{2}$ population, since identical top SNPs were observed at these loci. The multiple associations clearly indicate a pleiotropic role of these QTL in regulation of growth and fat deposition in pigs. In addition, SSC14 showed time-dependent effects on body weight at three distinct regions. Each region was associated with changes in body weight at a different time point (days 0, 21 or 240) in Sutai pigs.

\section{Discussion}

\section{GWAS versus traditional QTL mapping}

Compared to traditional QTL mapping approaches, one obvious advantage of GWAS is that it can use highdensity markers along the entire genome and thus capture enough LD (linkage disequilibrium) to identify a majority of potential causal variants. The GWAS top SNPs are usually in the vicinity of causal mutations, allowing us to more accurately pinpoint the most likely candidate genes for the loci of interest. In $F_{2}$ crosses, clusters of significant SNPs are usually revealed at the major QTL by long-range LD patterns. Further haplotypesharing analyses based on these significant SNPs can then be used to refine the location of the QTL. By using GWAS and haplotype-sharing analysis, we identified HMGA1 and PLAG1 as promising candidate genes at the prominent loci on SSC7 and SSC4, respectively. This finding has the potential to significantly benefit the ultimate characterization of the underlying variation in these loci in the near future.

We note that fewer significant loci were detected by GWAS in this study than in a previous study in which we used QTL mapping in the same White Duroc $x$ Erhualian $\mathrm{F}_{2}$ intercross population [28]. This inconsistency could be caused by two factors. First, GWAS employs a more stringent Bonferroni-corrected threshold. This conservative threshold reduces the false discovery rate but simultaneously decreases the ability to detect loci with moderate or small effects. Second, only additive SNP effects were included in the mixed linear GWAS model while dominant effects were also considered in the QTL model.

\section{Shared and unique loci in the $F_{2}$ and Sutai populations} In this study, we did not observe any locus of genomewide significance that was shared between the White Duroc $\times$ Erhualian $F_{2}$ intercross and Sutai populations, a finding that highlights the complex genetic architecture 
of growth and fatness traits. Sutai pigs were originally developed from a cross between Chinese Taihu pigs (including Erhualian and Meishan pigs) and Duroc pigs and have been artificially selected for at least 18 generations for lean meat and litter size [2]. In theory, some GWAS signals should be shared between the White Duroc $\times$ Erhualian $F_{2}$ intercross pigs and the Sutai pigs due to their similar genomic backgrounds. In fact, we have detected both shared and distinct GWAS results for multiple phenotypic traits in the two populations [55-57]. To test why the prominent loci associated with fatness and growth traits on SSC2, 4, 7 and $\mathrm{X}$ that were identified in the intercross pigs are absent from Sutai pigs, we further examined the most significant locus on SSC7. We found that the Q-bearing haplotype that corresponded to the critical region of this locus was not inherited by the current Sutai population from its Chinese Taihu founders; indeed, in the current Sutai population, all individuals are homozygous for the q-type haplotype. This fact partly explains why the SSC7 QTL effect has disappeared in Sutai pigs. However, we are unable to rule out other possibilities for certain. The molecular basis that underlies porcine growth and fatness traits could be more complicated than expected.

\section{Conclusions}

We performed a GWAS of 14 growth and eight fatness traits in a White Duroc $\times$ Erhualian $F_{2}$ intercross population and a Chinese Sutai half-sib population. Fourteen QTL of genome-wide significance and 39 suggestive loci of chromosome-wide significance were identified on 16 chromosomes. The discovery of strongly associated loci on SSC2, 4, 7 and X confirms our previous QTL mapping results. The critical region of the locus on SSC7 was refined to a $\sim 750 \mathrm{~kb}$ segment, and the $\mathrm{Q}$ allele that promotes fast growth and decreases fat deposition was confirmed to be of Chinese origin and probably arose after the domestication of Chinese wild boars. Population genetic analysis revealed that geographically diverse Chinese breeds segregate for the $Q$ allele, which illustrates the importance of this allele in genetic improvement of Chinese indigenous pigs. Several promising candidate genes were identified, including $H M G A 1$ at the SSC7 locus and PLAG1 at the SSC4 locus. No significant loci were shared between the Sutai and $F_{2}$ populations, and both time-constant and time-specific loci were detected in association with growth traits at different stages, which illustrates the complexity of the molecular mechanisms that underlie growth and fatness in pigs. Our findings provide novel insights into the genetic basis of growth and fatness in pigs and may contribute to the identification of the causal variants for the identified loci, especially for the major loci on SSC4 and SSC7.

\section{Additional files}

Additional file 1: Table S1. Descriptive statistics for growth and fatness traits in the tested samples. This table provides data on the descriptive statistics for growth and fatness traits in the tested samples.

Additional file 2: Table S2. Suggestive loci identified by GWAS for growth and fatness traits in White Duroc $\times$ Erhualian $F_{2}$ pigs and Sutai pigs. The table presents the loci of chromosome-wide significance that were identified by GWAS for growth and fatness traits in White Duroc $\times$ Erhualian $F_{2}$ pigs and Sutai pigs.

Additional file 3: Figure S1. Manhattan plots for the analyses of fatness traits in Sutai pigs. The GWAS for ABFT (A), LFW (B), VFW (C) and AFW (D) in Sutai pigs. In the Manhattan plots, negative $\log _{10} P$ values of the qualified SNPS were plotted against their genomic positions. The SNPS on different chromosomes are denoted by different colors. The solid and dashed lines indicate the 5\% genome-wide and chromosomewide (i.e., suggestive) Bonferroni-corrected thresholds, respectively. ABFT: average backfat thickness; LFW: leaf fat weight; VFW: veil fat weight; AFW: abdominal fat weight.

Additional file 4: Figure S2. Box-and-whisker plot for the effect of haplotypes corresponding to the 1.7-Mb critical region of the SSC7 locus on fat deposition in $\mathrm{F}_{2}$ animals. Phenotypic values of average backfat thickness (ABFT) and leaf fat weight (LFW) are on the $\mathrm{Y}$-axis. $\mathrm{N}$ indicates the number of each haplotype. Haplotype 1, from 13 Erhualian founder sows, is associated with decreased fat deposition and is thus defined as the Q-bearing haplotype. Haplotypes 2, 3, 4 and 5 are associated with increased fat deposition and thus considered to be the q-bearing haplotype. Haplotypes 2 and 3 were inherited from two White Duroc founder sires (73 and 75) and haplotype 4 from two Erhualian $F_{0}$ sows (124 and 126). Haplotype 5 is a recombinant haplotype between haplotypes 2 and 3. Haplotypes 2, 3 and 4 correspond to the q1, q2 and q3 chromosomes in Figure 3B, respectively. Haplotype 6 , which is indicated by "?", was inherited from Erhualian $F_{0}$ sows 142 and 146. Of the $930 \mathrm{~F}_{2}$ animals genotyped for $\sim 62000$ SNPs using the Illumina porcine $60 \mathrm{~K}$ DNA chip, only $10 \mathrm{~F}_{2}$ individuals carried this haplotype, and its QTL status could not be deduced (Panel A). Panel B shows the effect of this haplotype on average backfat thickness and leaf fat weight after the inclusion of seven additional individuals carrying the haplotype.

Additional file 5: Figure S3. Pedigree of $17 F_{2}$ and $F_{3}$ individuals carrying the $\mathrm{q}$ ? haplotype. All 17 individuals are indicated in green. Note that individuals 1881 and 2086 had not been recorded for fatness traits at slaughter since these individuals were used to produce $\mathrm{F}_{3}$ offspring.

Additional file 6: Table S3. Phenotypic differences in average backfat thickness and leaf fat weight among the Q, q and q? haplotypes. This table provides data that illustrate the phenotypic differences in average backfat thickness and leaf fat weight among the Q, q and q? haplotypes.

Additional file 7: Figure S4. Known and predicted protein-protein interactions of HMGA1 in the STRING database (available at http://stringdb.org). HMGA1 can bind with PPARG (peroxisome proliferator-activated receptor gamma), a key regulator of fat-cell differentiation and glucose homeostasis.

Additional file 8: Figure S5. Manhattan plots for the analyses of backfat thickness at the first rib in $F_{2}$ animals using the 60 K-chip SNPs, PPARD G32E and HMGA1 g.3135C > T on SSC7. In the Manhattan plots, negative $\log _{10} P$ values of the filtered high-quality SNPs were plotted against their genomic positions. PPARD G32E and HMGA1 g.3135C > T SNPS are highlighted in green and red, respectively.

Additional file 9: Figure S6. Manhattan plots for the analyses of fatness traits after accounting for the effect of the SSC7 locus in $F_{2}$ animals. Description: In the Manhattan plots, negative $\log _{10} P$ values of the filtered high-quality SNPs were plotted against their genomic positions after correcting for the effect of HMGA1 g.3135C > T. The SNPs on different chromosomes are denoted by different colors. The solid and dashed lines indicate the $5 \%$ genome-wide and chromosome-wide (i.e., suggestive) Bonferroni-corrected thresholds, respectively. ABFT: average backfat thickness; LFW: leaf fat weight; VFW: veil fat weight; AFW: abdominal fat weight. 


\section{Competing interests}

The authors declare that they have no competing interests.

\section{Authors' contributions}

JR and LH conceived and designed the experiment. RQ and JG performed the experiment. JR, ZZ, RQ and JG analyzed the data. LL, XX, YF, LC, HA and $J M$ contributed reagents/materials/analysis tools. JR and RQ wrote the paper. All authors read and approved the final manuscript.

\section{Acknowledgments}

This work was supported by funds from the Program for Changjiang Scholars and Innovative Research Team in University (IRT1136) to JR and from the National Natural Science Foundation of China (31060298) to JG.

\section{Received: 13 September 2014 Accepted: 8 January 2015}

\section{Published online: 14 March 2015}

\section{References}

1. Ramos-Onsins SE, Burgos-Paz W, Manunza A, Amills M. Mining the pig genome to investigate the domestication process. Heredity. 2014;113:471-84.

2. China National Commission of Animal Genetic Resource. Animal Genetic Resources in China: Pig. 1st ed. Beijing: China Agriculture Press; 2011.

3. Bidanel JP, Milan D, lannuccelli N, Amigues Y, Boscher MY, Bourgeois F, et al. Detection of quantitative trait loci for growth and fatness in pigs. Genet Sel Evol. 2001;33:289-309.

4. Walling GA, Visscher PM, Andersson L, Rothschild MF, Wang L, Moser G, et al. Combined analyses of data from quantitative trait loci mapping studies. Chromosome 4 effects on porcine growth and fatness. Genetics. 2000;155:1369-78.

5. Rohrer GA. Identification of quantitative trait loci affecting birth characters and accumulation of backfat and weight in a Meishan-White Composite resource population. J Anim Sci. 2000;78:2547-53.

6. Sato S, Oyamada Y, Atsuji K, Nade T, Kobayashi E, Mitsuhashi T, et al. Quantitative trait loci analysis for growth and carcass traits in a Meishan $\mathrm{x}$ Duroc F2 resource population. J Anim Sci. 2003;81:2938-49.

7. Van Laere AS, Nguyen M, Braunschweig M, Nezer C, Collette C, Moreau L, et al. A regulatory mutation in IGF2 causes a major QTL effect on muscle growth in the pig. Nature. 2003;425:832-6.

8. Fernandez Al, Perez-Montarelo D, Barragan C, Ramayo-Caldas Y, Ibanez-Escriche N, Castello A, et al. Genome-wide linkage analysis of QTL for growth and body composition employing the PorcineSNP60 BeadChip. BMC Genet. 2012;13:41

9. Gregersen VR, Conley LN, Sorensen KK, Guldbrandtsen B, Velander IH, Bendixen C. Genome-wide association scan and phased haplotype construction for quantitative trait loci affecting boar taint in three pig breeds. BMC Genomics. 2012;13:22.

10. Sahana G, Kadlecova V, Hornshoj H, Nielsen B, Christensen OF. A genomewide association scan in pig identifies novel regions associated with feed efficiency trait. J Anim Sci. 2013;91:1041-50.

11. Preissler R, Tetens J, Reiners K, Looft H, Kemper N. A genome-wide association study to detect genetic variation for postpartum dysgalactia syndrome in five commercial pig breeding lines. Anim Genet. 2013;44:502-8.

12. Okumura N, Matsumoto T, Hayashi T, Hirose K, Fukawa K, Itou T, et al. Genomic regions affecting backfat thickness and cannon bone circumference identified by genome-wide association study in a Duroc pig population. Anim Genet. 2013:44:454-7.

13. Guo $Y$, Mao H, Ren J, Yan X, Duan $Y$, Yang G, et al. A linkage map of the porcine genome from a large-scale White Duroc $x$ Erhualian resource population and evaluation of factors affecting recombination rates. Anim Genet. 2009;40:47-52.

14. Purcell S, Neale B, Todd-Brown K, Thomas L, Ferreira MA, Bender D, et al. PLINK: a tool set for whole-genome association and population-based linkage analyses. Am J Hum Genet. 2007:81:559-75.

15. Breslow NE, Clayton DG. Approximate inference in generalized linear mixed models. J Am Stat Assoc. 1993;88:9-25.

16. Bradbury PJ, Zhang Z, Kroon DE, Casstevens TM, Ramdoss Y, Buckler ES. TASSEL: software for association mapping of complex traits in diverse samples. Bioinformatics. 2007;23:2633-5.

17. Yu J, Pressoir G, Briggs WH, Vroh Bi I, Yamasaki M, Doebley JF, et al. A unified mixed-model method for association mapping that accounts for multiple levels of relatedness. Nat Genet. 2006;38:203-8.
18. Hayes BJ, Goddard M. Technical note: Prediction of breeding values using marker-derived relationship matrices. J Anim Sci. 2008:86:2089-92.

19. Eding $H$, Meuwissen THE. Marker-based estimates of between and within population kinships for the conservation of genetic diversity. J Anim Breed Genet. 2001;118:141-59.

20. Aulchenko YS, Ripke S, Isaacs A, Van Duijn CM. GenABEL: an R library for genome-wide association analysis. Bioinformatics. 2007;23:1294-6.

21. Aulchenko YS, De Koning DJ, Haley C. Genomewide rapid association using mixed model and regression: a fast and simple method for genomewide pedigree-based quantitative trait loci association analysis. Genetics. 2007;177:577-85.

22. Druet T, Georges M. A hidden Markov model combining linkage and linkage disequilibrium information for haplotype reconstruction and quantitative trait locus fine mapping. Genetics. 2010;184:789-98.

23. Zhang Z, Guillaume F, Sartelet A, Charlier C, Georges M, Farnir F, et al. Ancestral haplotype-based association mapping with generalized linear mixed models accounting for stratification. Bioinformatics. 2012;28:2467-73.

24. Druet T, Farnir FP. Modeling of identity-by-descent processes along a chromosome between haplotypes and their genotyped ancestors. Genetics. 2011;188:409-419.

25. Ai H, Huang L, Ren J. Genetic diversity, linkage disequilibrium and selection signatures in chinese and Western pigs revealed by genome-wide SNP markers. PLOS ONE. 2013;8:e56001.

26. Scheet P, Stephens M. A fast and flexible statistical model for large-scale population genotype data: applications to inferring missing genotypes and haplotypic phase. Am J Hum Genet. 2006;78:629-44.

27. Tamura K, Peterson D, Peterson N, Stecher G, Nei M, Kumar S. MEGA5: molecular evolutionary genetics analysis using maximum likelihood, evolutionary distance, and maximum parsimony methods. Mol Biol Evol. 2011;28:2731-9.

28. Ai H, Ren J, Zhang Z, Ma J, Guo Y, Yang B, et al. Detection of quantitative trait loci for growth- and fatness-related traits in a large-scale White Duroc $x$ Erhualian intercross pig population. Anim Genet. 2012;43:383-91.

29. de Koning DJ, Janss LL, Rattink AP, van Oers PA, de Vries BJ, Groenen MA, et al. Detection of quantitative trait loci for backfat thickness and intramuscular fat content in pigs (Sus scrofa). Genetics. 1999;152:1679-90.

30. Rohrer GA, Keele JW. Identification of quantitative trait loci affecting carcass composition in swine: I. Fat deposition traits. J Anim Sci. 1998;76:2247-54.

31. Wada Y, Akita T, Awata T, Furukawa T, Sugai N, Inage Y, et al. Quantitative trait loci (QTL) analysis in a Meishan x Gottingen cross population. Anim Genet. 2000;31:376-84.

32. Ren J, Duan Y, Qiao R, Yao F, Zhang Z, Yang B, et al. A missense mutation in PPARD causes a major QTL effect on ear size in pigs. PLoS Genet. 2011;7:e1002043.

33. Speliotes EK, Willer CJ, Berndt SI, Monda KL, Thorleifsson G, Jackson AU, et al. Association analyses of 249,796 individuals reveal 18 new loci associated with body mass index. Nat Genet. 2010;42:937-48.

34. Kim JJ, Lee HI, Park T, Kim K, Lee JE, Cho NH, et al. Identification of 15 loci influencing height in a Korean population. J Hum Genet. 2010;55:27-31.

35. liritano S, Chiefari E, Ventura V, Arcidiacono B, Possidente K, Nocera A, et al. The HMGA1-IGF-I/IGFBP system: a novel pathway for modulating glucose uptake. Mol Endocrinol. 2012;26:1578-89.

36. Gudbjartsson DF, Walters GB, Thorleifsson G, Stefansson H, Halldorsson BV Zusmanovich $\mathrm{P}$, et al. Many sequence variants affecting diversity of adult human height. Nat Genet. 2008;40:609-15.

37. Kubota N, Terauchi Y, Miki H, Tamemoto H, Yamauchi T, Komeda K, et al. PPAR gamma mediates high-fat diet-induced adipocyte hypertrophy and insulin resistance. Mol Cell. 1999;4:597-609.

38. Andersson L, Haley CS, Ellegren $H$, Knott SA, Johansson M, Andersson $K$, et al. Genetic mapping of quantitative trait loci for growth and fatness in pigs. Science. 1994;263:1771-4.

39. Perez-Enciso M, Clop A, Noguera JL, Ovilo C, Coll A, Folch JM, et al. A QTL on pig chromosome 4 affects fatty acid metabolism: evidence from an Iberian by Landrace intercross. J Anim Sci. 2000;78:2525-31.

40. Moller M, Berg F, Riquet J, Pomp D, Archibald A, Anderson S, et al High-resolution comparative mapping of pig Chromosome 4, emphasizing the FAT1 region. Mamm Genome. 2004;15:717-31.

41. Berg F, Stern S, Andersson K, Andersson L, Moller M. Refined localization of the FAT1 quantitative trait locus on pig chromosome 4 by marker-assisted backcrossing. BMC Genet. 2006;7:17. 
42. Mercade A, Estelle J, Noguera JL, Folch JM, Varona L, Silio L, et al. On growth, fatness, and form: a further look at porcine chromosome 4 in an Iberian x Landrace cross. Mamm Genome. 2005;16:374-82.

43. Mercade A, Perez-Enciso M, Varona L, Alves E, Noguera JL, Sanchez A, et al. Adipocyte fatty-acid binding protein is closely associated to the porcine FAT1 locus on chromosome 4. J Anim Sci. 2006;84:2907-13.

44. Karim L, Takeda H, Lin L, Druet T, Arias JA, Baurain D, et al. Variants modulating the expression of a chromosome domain encompassing PLAG1 influence bovine stature. Nat Genet. 2011;43:405-13.

45. Rubin CJ, Megens HJ, Martinez Barrio A, Maqbool K, Sayyab S, Schwochow $D$, et al. Strong signatures of selection in the domestic pig genome. Proc Natl Acad Sci U S A. 2012;109:19529-36.

46. Nezer C, Moreau L, Brouwers B, Coppieters W, Detilleux J, Hanset R, et al. An imprinted QTL with major effect on muscle mass and fat deposition maps to the IGF2 locus in pigs. Nat Genet. 1999;21:155-6.

47. Cepica S, Bartenschlager H, Geldermann H. Mapping of QTL on chromosome $X$ for fat deposition, muscling and growth traits in a wild boar $x$ Meishan F2 family using a high-density gene map. Anim Genet. 2007;38:634-8.

48. Cepica S, Masopust M, Knoll A, Bartenschlager H, Yerle M, Rohrer GA, et al. Linkage and $\mathrm{RH}$ mapping of 10 genes to a QTL region for fatness and muscling traits on pig chromosome X. Anim Genet. 2006:37:603-4.

49. Guo YM, Lee GJ, Archibald AL, Haley CS. Quantitative trait loci for production traits in pigs: a combined analysis of two Meishan $\mathrm{x}$ Large White populations. Anim Genet. 2008;39:486-95.

50. Milan D, Bidanel JP, lannuccelli N, Riquet J, Amigues Y, Gruand J, et al. Detection of quantitative trait loci for carcass composition traits in pigs. Genet Sel Evol. 2002;34:705-28.

51. Ma J, lannuccelli N, Duan Y, Huang W, Guo B, Riquet J, et al Recombinational landscape of porcine $X$ chromosome and individual variation in female meiotic recombination associated with haplotypes of Chinese pigs. BMC Genomics. 2010;11:159.

52. Oka S, Masutani H, Liu W, Horita H, Wang D, Kizaka-Kondoh S, et al. Thioredoxin-binding protein-2-like inducible membrane protein is a novel vitamin D3 and peroxisome proliferator-activated receptor (PPAR)gamma ligand target protein that regulates PPARgamma signaling. Endocrinology. 2006;147:733-43.

53. Oka S, Yoshihara E, Bizen-Abe A, Liu W, Watanabe M, Yodoi J, et al. Thioredoxin binding protein-2/thioredoxin-interacting protein is a critical regulator of insulin secretion and peroxisome proliferator-activated receptor function. Endocrinology. 2009;150:1225-34.

54. Cherel P, Pires J, Glenisson J, Milan D, lannuccelli N, Herault F, et al. Joint analysis of quantitative trait loci and major-effect causative mutations affecting meat quality and carcass composition traits in pigs. BMC Genet. 2011;12:76.

55. Yang B, Zhang W, Zhang Z, Fan Y, Xie X, Ai H, et al. Genome-wide association analyses for fatty acid composition in porcine muscle and abdominal fat tissues. PLoS ONE. 2013:8:e65554.

56. Ma J, Yang J, Zhou L, Zhang Z, Ma H, Xie X, et al. Genome-wide association study of meat quality traits in a White Duroc $x$ Erhualian F2 intercross and Chinese Sutai pigs. PLoS ONE. 2013;8:e64047.

57. Zhang Z, Hong Y, Gao J, Xiao S, Ma J, Zhang W, et al. Genome-wide association study reveals constant and specific loci for hematological traits at three time stages in a White Duroc x Erhualian F2 resource population. PLOS ONE. 2013:8:e63665.

\section{Submit your next manuscript to BioMed Central and take full advantage of:}

- Convenient online submission

- Thorough peer review

- No space constraints or color figure charges

- Immediate publication on acceptance

- Inclusion in PubMed, CAS, Scopus and Google Scholar

- Research which is freely available for redistribution 